\title{
HEDEOMA TEYUCUARENSIS (LAMIACEAE), UNA ESPECIE NUEVA DE MISIONES, ARGENTINA
}

\author{
Héctor A. Keller \& Sara G. Tressens
}

Instituto de Botánica del Nordeste, UNNE-CONICET, Casilla de Correo 209, 3400 Corrientes, Corrientes, Argentina; kellerhector@hotmail.com (autor corresponsal).

\begin{abstract}
Keller, H. A. \& S. G. Tressens. 2016. Hedeoma teyucuarensis (Lamiaceae), a new species from Misiones, Argentina. Darwiniana, nueva serie 4(1): 5-11.

A new species of Hedeoma (Lamiaceae) is described based on samples collected in Misiones Province, Argentina. A botanical illustration, photos of the new taxon, information about its habitat, status of conservation, and importance for local people is also provided, including a key to the species of Hedeoma from the Southern Cone of America.
\end{abstract}

Keywords. Aromatic plants; ethnobotany; Flora Argentina; Hedeoma; new taxon.

Resumen. Keller, H. A. \& S. G. Tressens. 2016. Hedeoma teyucuarensis (Lamiaceae), una especie nueva de Misiones, Argentina. Darwiniana, nueva serie 4(1): 5-11.

Se describe una nueva especie de Hedeoma (Lamiaceae) sobre la base de muestras recolectadas en la provincia de Misiones, Argentina. Se incluye una ilustración, fotos del nuevo taxon, información acerca de su hábitat, estado de conservación e importancia para los pobladores locales, junto con una clave que permite diferenciar las especies de Hedeoma del Cono Sur de América.

Palabras clave. Etnobotánica, Flora Argentina; Hedeoma; nuevo taxón; plantas aromáticas.

\section{INTRODUCCIÓN}

Un estudio etnobotánico llevado a cabo entre los años 2008/2010 en el distrito de los campos de la provincia de Misiones permitió obtener información sobre los usos de una planta aromática conocida por los pobladores aledaños a los pastizales del paraje Teyú Cuaré como "mentita campera" o "poleo de campo". Durante las campañas realizadas en el marco de dicha investigación se recolectó una muestra de herbario de la misma, con muchos frutos y algunas flores. Recientemente, se reco- lectaron dos muestras de la planta con abundantes flores, permitiendo su identificación a nivel de género. No obstante la combinación de diversos atributos morfológicos de las muestras no coinciden con las especies conocidas motivando la redacción del presente trabajo el cual reconoce una especie nueva.

El género Hedeoma Pers. (Lamiaceae) cuenta con cerca de medio centenar de especies que en su mayor parte se distribuyen en América del Norte, principalmente México y Estados Unidos; y sólo cinco especies, en América del Sur (Irving, 1980, 
Slanis \& Bulacio, 2005). Aunque Persoon (1807) al establecer el género no ofrece una elucidación etimológica de su propuesta, la expresión Hedeoma (del latín hedeo: heder) seguramente alude a las virtudes aromáticas de sus especies. Además de haber inspirado epítetos específicos como $\mathrm{pi}$ perita, pulegioides, thymoides, en la Argentina y en México las cualidades odoríferas también se reflejan en las denominaciones vernáculas de varios representantes del género, las que incluyen en su conformación expresiones asociativas como por ejemplo "comino", "menta", "orégano", "peperina", "poleo" y "tomillo" (Santi \& Retamar, 1983; Casillas Alcalá, 1992; Del Vitto et al., 1997; Martínez, 2005; Slanis \& Bulacio, 2005; Dadé et al., 2009; Viveros-Valdez et al., 2010).

Para el Cono Sur se han documentado solamente cuatro especies de Hedeoma: H. multiflora Benth. que crece en el centro y sur de la Argentina, en el estado de Rio Grande do Sul de Brasil, en la Región IV, V y VII de Chile y en el depto. Paysandú de Uruguay; $H$. medium Epling en las provincias de Entre Ríos y Buenos Aires (Argentina) y los departamentos Paysandú y Soriano (Uruguay); $H$. mandoniana Wedd. en el Noroeste de la Argentina y H. polygalifolia Benth. en el estado de Rio Grande do Sul, Brasil (Harley, 2008).

En la presente contribución se describe e ilustra una nueva especie de Hedeoma, H. teyucuarensis, lo que eleva a cinco el número de especies registradas para el Cono Sur, cuatro de las cuales crecen en la Argentina. Se trata asimismo de la primera cita del género para la provincia de Misiones. Al tratarse de un recurso florístico empleado por pobladores se brinda información sobre sus usos, así como también sobre la situación de conservación de la especie y del hábitat que la misma comparte con otros endemismos.

\section{TRATAMIENTO TAXONÓMICO}

Hedeoma teyucuarensis H. A. Keller \& Tressens, sp. nov. TIPO: Argentina, Misiones, San Ignacio, cercanías de aldea aborigen Pindoty, 15-X-2009 (fl, fr), H. A. Keller \& J. J. Araujo 7559 (holotipo CTES[0040321]). Figs. 1 y 2.

Suffrutex ad $65 \mathrm{~cm}$ altum. Folia sessilia aut subsessilia, 15-35 mm longa, 5-13 mm lata. Inflorescentia axillaris 3-20 flora. Calyx 60-65 mm longus; annulus (carpostegium) prope apicem tubi situs. Corolla ad 7,5 mm longam.

Sufrútice aromático de hasta $65 \mathrm{~cm}$ alt.; ramas, hojas y flores punteado-glandulosas, con indumento de pelos simples de diferente longitud. Ramas erectas, con indumento denso de pelos retrorsos, glabrescentes en la base. Entrenudos de 0,5-10 $\mathrm{cm}$. Hojas sésiles o con un brevísimo pecíolo ca. 0,5 mm. Lámina de 1,5-3,5 x 0,5-1,3 cm, elípticas, obovadas, margen entero o brevemente denticulado en la mitad apical; ápice agudo, base atenuada, epi- e hipofilo con indumento denso. Inflorescencias axilares cimosas, congestas, 3-20 floras, agregadas en pseudoespigas terminales de hasta $7 \mathrm{~cm}$. Pedúnculos ca. $1 \mathrm{~mm}$., con pelos retrorsos. Brácteas y bractéolas hirtelas por fuera, glabras por dentro, margen ciliado; brácteas ovadas o elípticas de 2-3 mm, bractéolas elípticas o subuladas de 1-2,5 mm. Pedicelos de 1,5-2 $\mathrm{mm}$, cubiertos de pelos retrorsos. Cáliz de 6-6,5 $\mathrm{mm}$, tubular, bilabiado, velloso sobre las venas, dientes densamente ciliados en el margen; tubo de 3,5-4,5 $\mathrm{mm}$, levemente comprimido a la altura del carpostegio, interior del tubo con carpostegio denso, ca. 0,8 $\mathrm{mm}$ alt., en el 1/4 apical, el resto de la superficie interna del tubo glabro; labio superior de 1-1,2 mm, dientes triangulares, erectos, connados en la mitad inferior; labio inferior de 2-2,2 $\mathrm{mm}$, dientes angostamente triangulares, levemente incurvos. Corola blanca con máculas violáceas en el labio inferior. Tubo corolino de 6-7,5 mm, infundibuliforme, velloso por fuera excepto en la porción inferior que rodea a las clusas donde es papiloso; por dentro glabro a la altura de las clusas, mitad inferior con pelos breves y rectos que se prolongan hacia el labio inferior en dos franjas ubicadas entre los lóbulos laterales y el lóbulo central; labios vellosobarbados por fuera y papilosos por dentro; labio superior levemente emarginado, ca. 3 × $2 \mathrm{~mm}$, la zona media, pubescente y ligeramente cóncava, rodea a los estambres y al estilo; labio inferior de $5,5 \times 5 \mathrm{~mm}$, subvelloso entre los lóbulos laterales, lóbulo central de $3 \times 4 \mathrm{~mm}$, obovado, ápice emarginado, base atenuada, margen crenado, lóbulos laterales menores. Estambres inferiores 


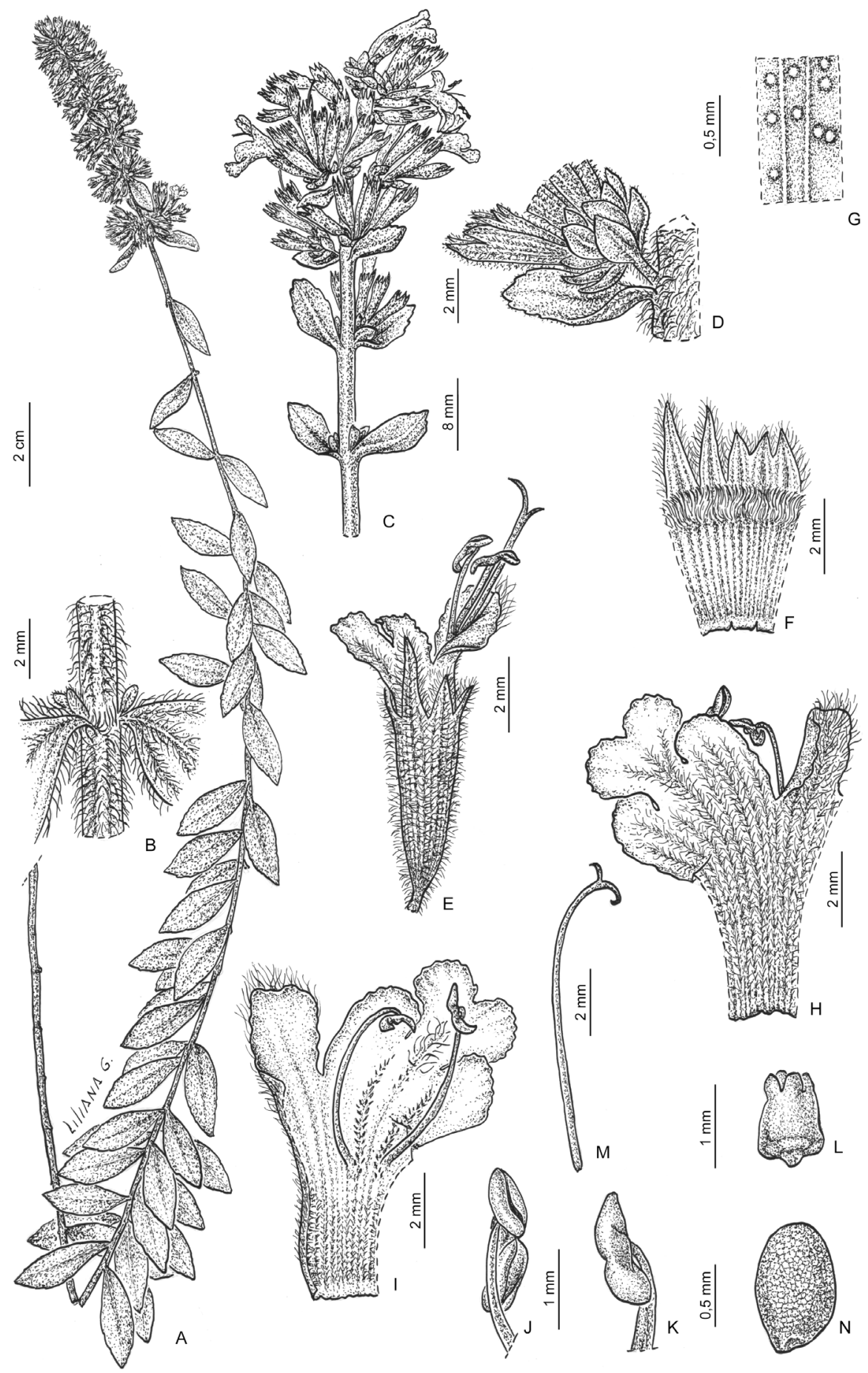

Fig. 1. Hedeoma teyucuarensis. A, rama fértil. B, indumento del tallo. C, detalle de la inflorescencia. D, címula. E, flor. $\mathbf{F}$, superficie interna del cáliz. G, estructuras glandulares del cáliz. H, corola, superficie externa. I, corola y estambres. J, K, anteras. L, ovario. M, estilo y estigma. N, clusa. A, B y N, de Keller \& Araujo 7759 (CTES). C-M, de Keller \& Ramírez 12919 (CTES). 

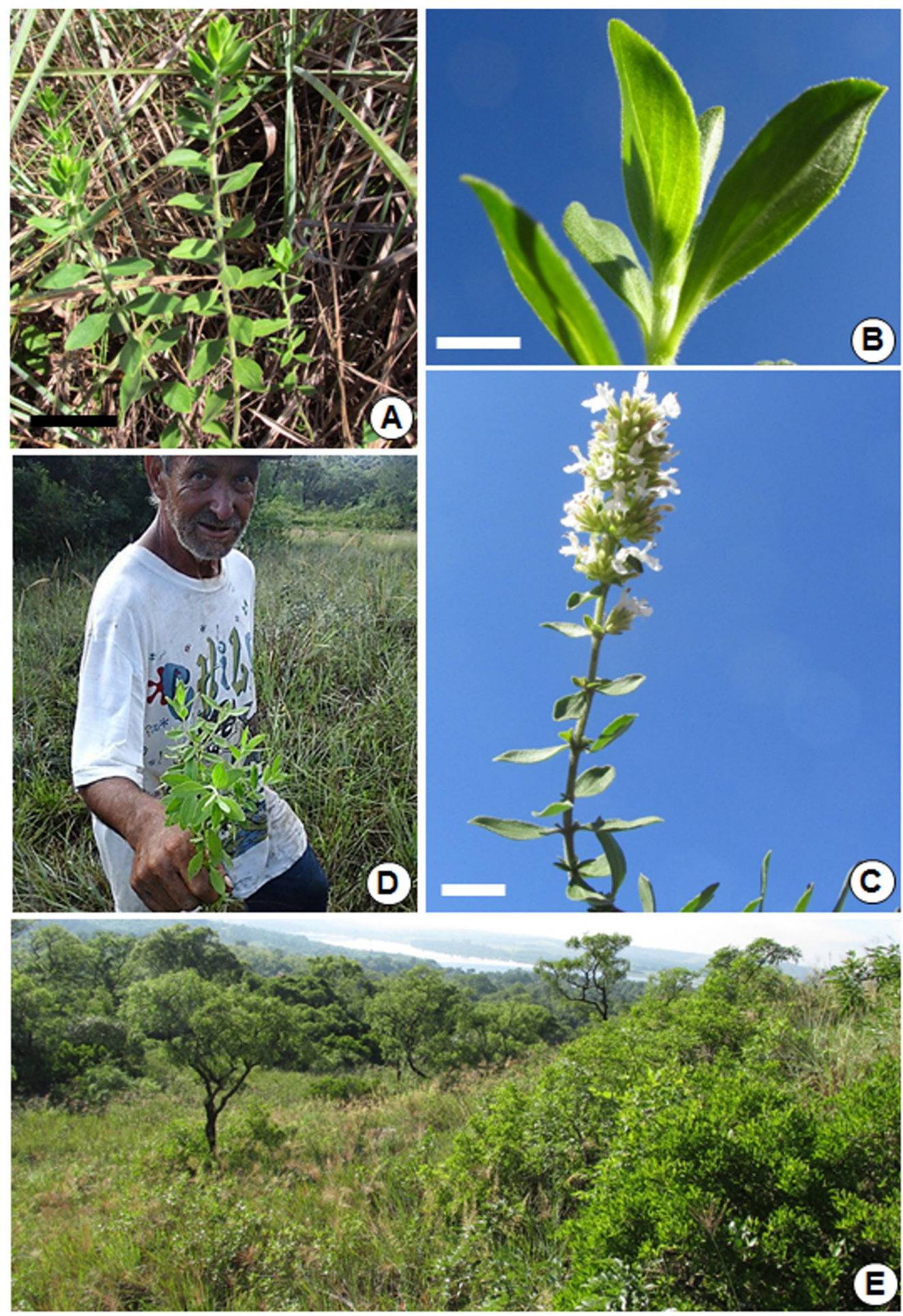

Fig. 2. Hedeoma teyucuarensis. A, planta. B, ápice vegetativo. C, rama florífera. D, recolección para su empleo en la farmacopea local. E, ambiente. Figura en color en la versión en línea http://www.ojs.darwin. edu.ar/index.php/darwiniana/article/view/692/674 
insertos $\pm 2,5 \mathrm{~mm}$ por debajo del labio inferior, filamentos de $\pm 5 \mathrm{~mm}$, glabros, tecas divaricadas. Estilo de $\pm 9 \mathrm{~mm}$, bífido, segmento abaxial más largo que el adaxial. Clusas marrones de \pm 1 $\mathrm{mm}$, ligeramente ovoideas, superficie areolada, mucilaginosas al humedecerse.

Etimología. El epíteto específico hace referencia a la localidad típica de la especie, Teyú Cuaré, San Ignacio, Misiones, Argentina.

La especie es conocida y utilizada por pobladores del paraje Teyú Cuaré, quienes para nombrarla utilizan los siguientes fitónimos: "mentita", "mentita de campo", "mentita campera" y "poleo de campo". Sus ramas vegetativas y reproductivas son utilizadas como aromatizantes de la infusión de yerba mate y también del "tereré", una bebida refrescante. Para estos usos se recolecta el material en campo (Fig. 2D) o bien se suelen cultivar ejemplares en el ámbito hogareño. La decocción de sus raíces se emplea para combatir parásitos intestinales, para ello se hace ayuno durante medio día y se ingiere previamente una cucharada de azúcar con el fin de "congregar" a los parásitos que se desea exterminar. Este uso implica la extracción de la planta entera y causa alto impacto en la población de una especie que se encuentra en peligro crítico.

Distribución y hábitat. Conocida hasta el presente sólo para la localidad del holotipo (Fig. 3), donde se han hallado unos pocos ejemplares creciendo en un pastizal arbolado sobre un sustrato edáfico arenoso. En Misiones estos afloramientos de arenisca configuran un paisaje ondulado que geomorfológicamente responde a la continuación de la Sierra de Amambay de Paraguay y que desde el punto de vista florístico presentan elementos comunes con el Bioma Cerrado (Martínez Crovetto, 1963; Teruggi, 1970; Chébez, 1996; Biganzoli \& Múlgura de Romero, 2004; Velazco, 2014; Keller, 2015). Los tallos de Hedeoma teyucuarensis se yerguen entre una maraña irregular de gramíneas altas entre las que prevalece Axonopus suffultus (J.C. Mikan ex Trin.) Parodi. La vegetación leñosa es rala y consiste en arbustos esparcidos (Fig. 2E), principalmente Cordiera concolor (Cham.) Kuntze (Rubiaceae) y ejem- plares arbóreos bajos y tortuosos de Acosmium subelegans (Mohlenbr.) Yakovlev (Fabaceae) y Qualea cordata (Mart.) Spreng. (Vochysiaceae). Florece en septiembre-octubre y fructifica en octubre-noviembre.

Hedeoma teyucuarensis comparte la localidad, paraje Teyú Cuaré (San Ignacio, Misiones), con otras cinco especies endémicas de Misiones: Austrochthamalia teyucuarensis H. A. Keller (Apocynaceae), Hyptis australis Epling (Lamiacee), Mesosetum comatum Swallen (Poaceae), Oxypetalum teyucuarense Farinaccio \& Keller (Apocynaceae) y Lessingianthus teyucuarensis (Cabrera) H. Rob. (Asteracee). El hallazgo de este endemismo refuerza los argumentos expuestos por Fontana (2005) para ampliar el área protegida por el parque provincial Teyú Cuaré hasta los sitios donde además de los endemismos mencionados crecen muchas otras especies con alto valor de conservación.

En virtud de su singular vegetación, desde hace varias décadas la localidad es frecuentada por botánicos, fitosociólogos y coleccionistas de plantas, argentinos y extranjeros, y entre los años 2014-2015 ha sido objeto de un estudio florístico exhaustivo. No obstante ello, hasta el presente sólo se ha hallado una población de Hedeoma teyucuarensis, la que cuenta con 13 ejemplares distribuidos en un área menor a 0,1 ha, en un predio no protegido por leyes ambientales. Siguiendo cualquiera de los criterios establecidos por IUCN (2001), la especie puede ser considerada en peligro crítico y su extinción podrá evitarse solamente si se establecen programas de conservación in situ y ex situ.

Observaciones. De acuerdo con Irving (1980) Hedeoma Pers. es un género afín a Cunila D. Royen ex L. y Glechon Spreng. Sin embargo, el cáliz y la corola ambos marcadamente bilabiados en la especie que aquí se describe son caracteres que lo diferencian del género Cunila D. Royen ex L. Por su parte el número elevado de flores por inflorescencia (hasta 20), el cáliz con dientes desiguales y los estambres con filamentos de base no geniculada son caracteres que permiten distinguir a $\mathrm{He}$ deoma teyucuarensis de las especies del género Glechon Spreng.

La combinación de varios atributos 
morfológicos permite vincular a la especie aquí descripta con Hedeoma subgénero Saturejoides Irving (1980), pues se trata de un sufrútice perenne, el cáliz mide entre 6 y $6,5 \mathrm{~cm}$ long. y presenta dientes superiores claramente más cortos que los inferiores; las clusas son ovoides $\mathrm{y}$ se tornan mucilaginosas al mojarlas. En América del Sur este subgénero está representado por Hedeoma medium, $H$. multiflora y $H$. polygalifolia, todas plantas de menor altura con inflorescencias con hasta seis flores. De estas tres especies, $H$. polygalifolia es la única que presenta las inflorescencias axilares agrupadas en el ápice de las ramas, como ocurre en $H$. teyucuarensis, sin embargo, según Irving (1980), sus entrenudos son notablemente breves, ca. $1 \mathrm{~mm}$.

\section{Material adicional}

ARGENTINA. Misiones. Depto. San Ignacio, Paraje Teyú Cuaré, $27^{\circ} 16^{\prime}$ 52,9' S - 55 33' 43,5" W. 09-IX-2015 (fl), H. A. Keller \& R. E. Ramírez 12919 (CTES, SI); camino superior de entrada al Parque Teyú Cuaré, 22-IX-1997, Zuloaga 6456 (SI).

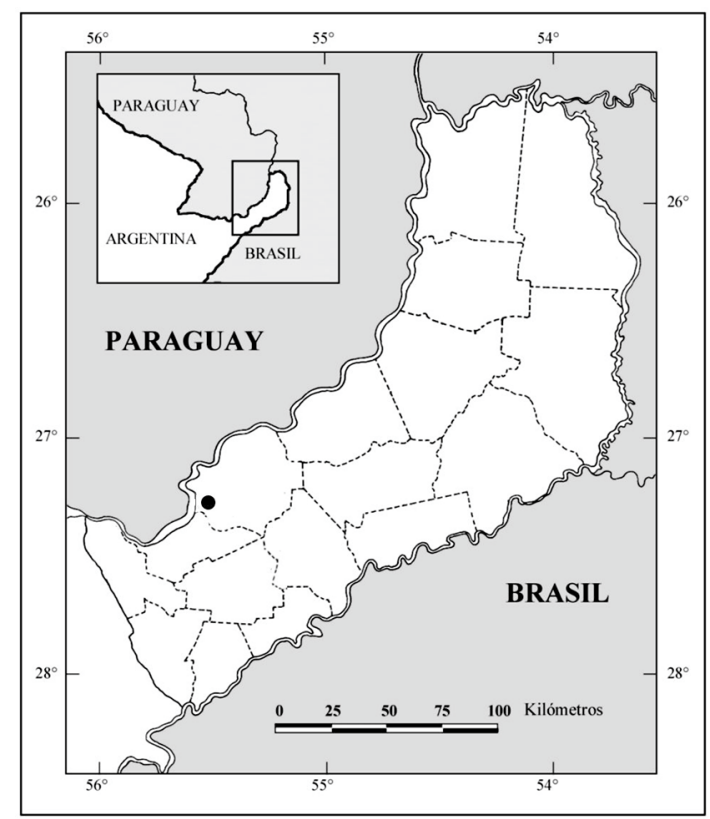

Fig. 3. Mapa de localización de la única población conocida de Hedeoma teyucuarensis.

\section{Clave de las especies de Hedeoma presentes en el Cono Sur de América del Sur}

1. Plantas de hasta $60 \mathrm{~cm}$ alt. Hojas de $15-35$ x 5-13 mm. Inflorescencias axilares 3-20 floras

H. teyucuarensis

1. Plantas de hasta $30 \mathrm{~cm}$ alt. Hojas de 5-17 x 1-7 mm. Inflorescencias axilares 1-6 floras

2(1'). Hojas ovadas, obovadas o elípticas; margen crenado en la mitad superior.

H. mandoniana

2. Hojas linear lanceoladas, linear elípticas, oblanceoladas u oblongas; margen entero 3

3(2'). Hierbas hasta de $10 \mathrm{~cm}$ alt., no cespitosas; tallos decumbentes con nudos enraizantes. Inflorescencias axilares 1-3 floras. Clusas esferoidales, superficie areolada H. polygalifolia

3. Hierbas hasta de $30 \mathrm{~cm}$ alt., cespitosas; tallos ascendentes. Inflorescencias axilares 1-6 floras. Clusas oblongas, superficie foveolada 4

4. Cáliz de 5-7 mm. Corola de 7-11 mm H. multiflora

4. Cáliz de 4,2-5 mm. Corola de 4-8 mm H. medium

\section{AGRADECIMIENTOS}

Las campañas que permitieron hallar y estudiar la población de la especie fueron financiadas por el Consejo Nacional de Investigaciones Científicas y Tecnológicas y por la Fundación Temaiken. Agradecemos a los pobladores locales por llevarnos hasta el sitio de hallazgo y por brindarnos información sobre la etnobotánica de la especie. Agradecemos las sugerencias de Nataly O'Leary del Instituto Darwinion. Expresamos nuestro agradecimiento a la dibujante Liliana Gómez por la ilustración de la especie. Durante las campañas se contó con el apoyo logístico de María Paula Bertolini, Jorge Néstor y Santiago Haddad y con la colaboración de Renzo Ramírez y de Jorge Araujo. 


\section{BIBLIOGRAFÍA}

Biganzoli, F. \& M. E. Múlgura de Romero. 2004. Inventario florístico del Parque Provincial Teyú Cuaré y alrededores (Misiones, Argentina). Darwiniana 42(1-4): 1-24.

Casillas-Alcalá, C. 1992. El orégano en México: panorama del primer exportador mundial. Huella 22: 7-43.

Chebez, J. C. 1996. Misiones Ñú. Campos Misioneros, algo más que el confín de la selva. Nuestras aves, Asociación Ornitológica del Plata 34: 4-16.

Dadé, M. M.; D. E. Fioravanti, G. R. Schinella \& H. A. Tournier 2009. Total antioxidant capacity and polyphenol content of 21 aqueous extracts obtained from native plants of Traslasierra valley (Argentina). Boletín Latinoamericano y del Caribe Plantas Medicinales y Aromáticas 8(6): 529-539.

Del Vitto, L. A.; E. M. Petenatti, \& M. E. Petenatti. 1997. Recursos Herbolarios de San Luis (República Argentina). Primera Parte: Plantas Nativas. Multequina 6: 49-66.

Fontana, J. L. 2005. Una propuesta para la conservación de los pajonales del Diplothemio-Axonopodetum. San Ignacio, Provincia de Misiones (Argentina). Facena 21: 55-67.

Harley, R. 2008. Lamiaceae, en F. O. Zuloaga, O. Morrone \& M. J. Belgrano (eds.), Catálogo de las Plantas Vasculares del Cono Sur (Argentina, Sur de Brasil, Chile, paraguay y Uruguay). Monographs in Systematic Botany from the Missouri Botanical Garden 107: 2362-2392.

Irving, R. S. 1980. The systematics of Hedeoma (Labiatae). Sida 8(3): 218-295.

IUCN. 2001. The IUCN red list of threatened species, version 2001.4. IUCN Red List Unit, Cambridge U.K. Available from: http://www.iucnredlist.org/ (accessed 11 March 2014).
Keller, H. A. 2015. Austrochthamalia teyucuarensis (Apocynaceae: Asclepiadoideae), una nueva especie endémica de Misiones, Argentina. Lilloa 52(1): 40-45.

Martínez, G. J. 2005. Recolección y Comercialización de Plantas Medicinales en el Departamento de Santa María, Provincia de Córdoba, Argentina. Acta Farmacéutica Bonaerense 24(4): 575-84.

Martínez Crovetto, R. N. 1963. Esquema fitogeográfico de la Provincia de Misiones (República Argentina). Bonplandia 1: $171-223$

Persoon, C. H. 1807. Synopsis Plantarum, seu enchiridium botanicum, complectens enumerationem systematicam specierum hucusque cognitarum, vol. 2. Paris. Bibliopolas Treuttel et Wurtz, Tübingen: J. G. Cottam.

Slanis, A. C. \& E. del V. Bulacio. 2005. Hedeoma mandonianum (Lamiaceae) en Argentina. Boletín de la Sociedad Argentina de Botánica 40(3-4): 285-287.

Santi, M. N. \& J. A. Retamar. 1983. Aceite esencial de Hedeoma multiflorum. Essenze e Derivati Agrumari 51(4): 353-359.

Teruggi, M. E. 1970. Bosquejo Geológico del Paraguay y la Provincia de Corrientes. Boletín de la Sociedad Argentina de Botánica 11, suplemento: 1-15.

Velazco, S. J. E. 2014. Estudo floristico e fitossociológico de três comunidades vegetáis em San Ignacio, provincia de Misiones, Argentina. Dissertação apresentada ao Curso de Pós-graduação em Engenharia Florestal do Setor de Ciências Agrarias da Universidade Federal do Parana, Curitiba, 180 pp.

Viveros-Valdez E., C. Rivas-Morales, A. Oranday-Cárdenas, J. Castro-Garza \& P. Carranza-Rosales. 2010. Antiproliferative effect from the Mexican poleo (Hedeoma drummondii). Journal of Medicinal Food 13(3):740-742. DOI: http://dx. doi.org/10.1089/jmf.2009.0041 